Supplement of Hydrol. Earth Syst. Sci., 25, 1333-1345, 2021

https://doi.org/10.5194/hess-25-1333-2021-supplement

(c) Author(s) 2021. CC BY 4.0 License.

(c) (i)

Supplement of

\title{
Hydrologic regimes drive nitrate export behavior in human-impacted watersheds
}

\author{
Galen Gorski and Margaret A. Zimmer \\ Correspondence to: Galen Gorski (ggorski@ucsc.edu)
}

The copyright of individual parts of the supplement might differ from the article licence. 


\section{S1 Calibration of nitrate probes}

For detailed QA/QC protocol see (Garrett, 2019) for USGS sites and (Jones et al., 2018) for IIHR sites. Instruments were calibrated for bias and sensor drift before deployment and during periodic site visits in which hand samples were collected and analyzed to check the sensor accuracy. For USGS sites, a difference of $0.3 \mathrm{mg} / \mathrm{L}$ or $5 \%$ between consecutive hand samples and sensor data triggered a sensor recalibration, but hand samples show good agreement with sensor data (Pellerin et al., 2014; Zimmer et al., 2019). Hach instruments measure concentrations of $\mathrm{NO}_{3}{ }^{-}$and $\mathrm{NO}_{2}{ }^{-}$due to the strong absorbance of $\mathrm{NO}_{2}{ }^{-}$between $210-220 \mathrm{~nm}$, however, $\mathrm{NO}_{2}{ }^{-}$ concentrations are generally very low in surface water and therefore sensor measurements are referred to as $\mathrm{NO}_{3}^{-}$.

\section{S2 Comparison of nitrate export loads to other studies}

Annual $\mathrm{NO}_{3}{ }^{-}$export loads compare favorably with estimates by (Jones et al., 2018), who estimated $\mathrm{NO}_{3}{ }^{-}$load at 35 sites across Iowa during 2016 including all five sites analyzed here using linear interpolation to fill data gaps. Our results show $\geq 90 \%$ agreement in annual load across all five sites with no systematic under or over prediction. Our four-year average annual loads are somewhat higher than those estimated by (Jones et al., 2018) for the period 1987-2016, likely due to higher than average discharge over the four-year period studied. 

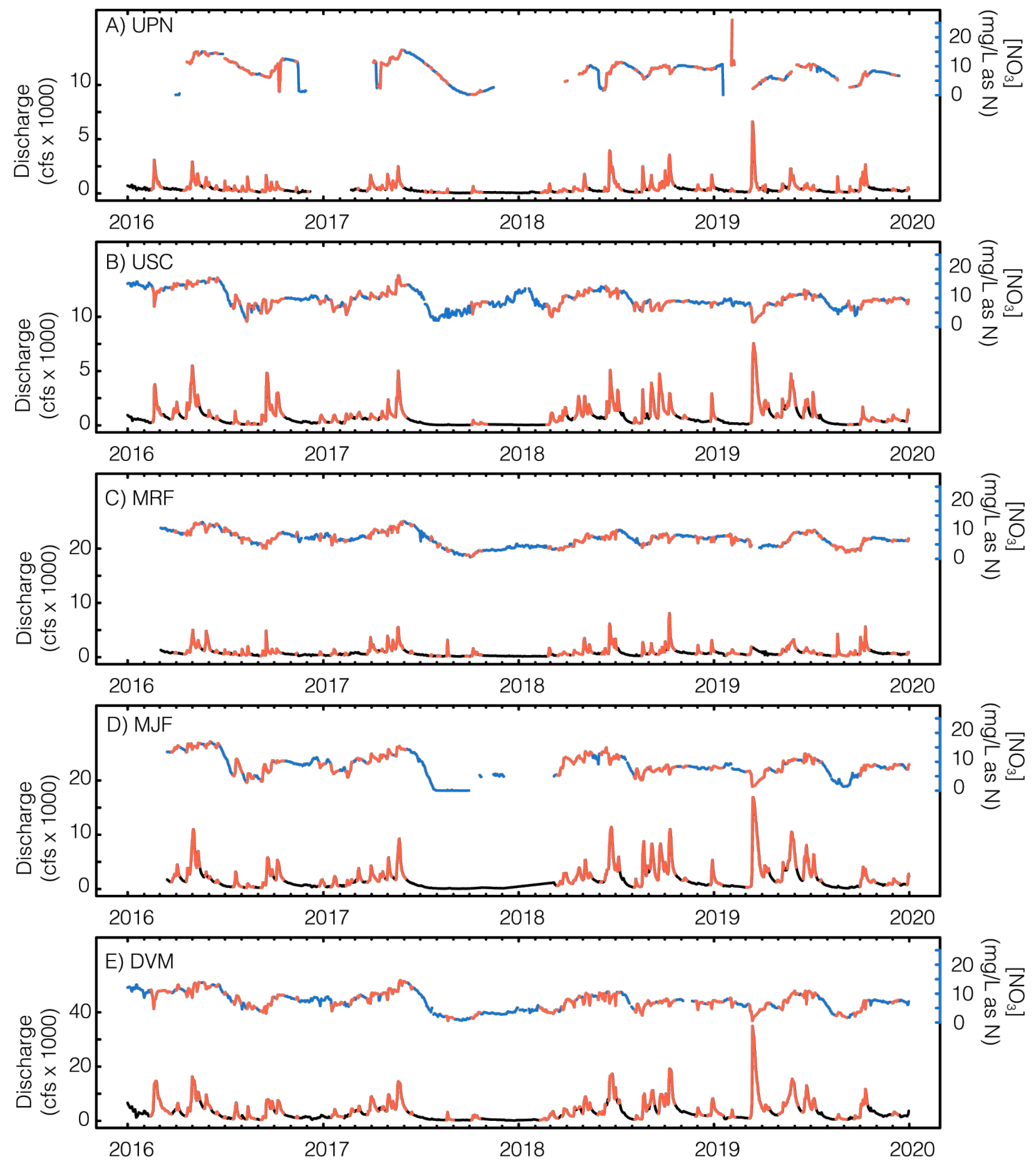

- Stormflow 二 Baseflow

Figure S1 Paired hydrographs and chemographs for the 5 study watersheds with identified events shown in red and baseflow shown in black (hydrograph) and blue (chemograph). Events were identified using the hydrograph, see section 2.3 for details. The record begins in January 2016 and ends in December 2019. 
Table S1 - Data coverage by watershed

\begin{tabular}{cccccc} 
Watershed & $\begin{array}{c}\text { Record } \\
\text { start date }\end{array}$ & $\begin{array}{c}\text { Record } \\
\text { end date }\end{array}$ & $\mathrm{n} \mathrm{obs}^{\mathrm{a}}$ & $\begin{array}{c}\text { coverage }^{\mathrm{b}} \\
(\%)\end{array}$ & $\begin{array}{c}\text { largest gap } \\
\text { (days) }\end{array}$ \\
\hline & & & & 71.8 & 127 \\
$U P N$ & $3 / 31 / 16$ & $12 / 13 / 19$ & 971 & 99.4 & 7 \\
MRF & $1 / 1 / 16$ & $12 / 31 / 19$ & 1451 & 91.9 & 15 \\
$M J F$ & $3 / 3 / 16$ & $12 / 31 / 19$ & 1344 & 88.7 & 94 \\
$D V M$ & $3 / 15 / 16$ & $12 / 31 / 19$ & 1229 & 16 \\
\hline
\end{tabular}

${ }^{a}$ Number of paired observations with discharge and $\left[\mathrm{NO}_{3}{ }^{-}\right]$

${ }^{\mathrm{b}}$ Data coverage for discharge was complete for all stations, gaps were $\left[\mathrm{NO}_{3}{ }^{-}\right]$non-reporting

Table S2 - Watershed land cover characteristics

\begin{tabular}{|c|c|c|c|c|c|c|c|c|c|c|}
\hline \multirow[b]{2}{*}{ Watershed } & \multirow[b]{2}{*}{ Area } & \multicolumn{2}{|c|}{ Water } & \multicolumn{2}{|c|}{ Developed } & \multicolumn{2}{|c|}{ Forest } & \multicolumn{2}{|c|}{ Crop } & \multirow[b]{2}{*}{$\begin{array}{l}\text { Drainage } \\
\text { infrastructure } \\
\text { density }\end{array}$} \\
\hline & & Total & $\begin{array}{c}100 \mathrm{~m} \\
\text { buffer } \\
\text { of } \\
\text { stream }\end{array}$ & Total & $\begin{array}{c}100 \mathrm{~m} \\
\text { buffer } \\
\text { of } \\
\text { stream }\end{array}$ & Total & $\begin{array}{c}100 \mathrm{~m} \\
\text { buffer } \\
\text { of } \\
\text { stream }\end{array}$ & Total & $\begin{array}{c}100 \mathrm{~m} \\
\text { buffer } \\
\text { of } \\
\text { stream }\end{array}$ & \\
\hline & $\left(\mathrm{km}^{2}\right)$ & $(\%)$ & $(\%)$ & $(\%)$ & $(\%)$ & $(\%)$ & $(\%)$ & $(\%)$ & $(\%)$ & $\left(\mathrm{km} / \mathrm{km}^{2}\right)$ \\
\hline$U P N$ & 1116 & 2 & 45 & 6 & 4 & 5 & 19 & 87 & 32 & 0.71 \\
\hline USC & 1840 & 2 & 39 & 5 & 5 & 1 & 16 & 92 & 39 & 1.11 \\
\hline$M R F$ & 2548 & 2 & 46 & 5 & 3 & 8 & 20 & 85 & 31 & 0.37 \\
\hline$M J F$ & 4188 & 2 & 47 & 5 & 3 & 2 & 15 & 91 & 35 & 0.93 \\
\hline$D V M$ & 8870 & 2 & 52 & 5 & 3 & 4 & 16 & 88 & 28 & 0.70 \\
\hline
\end{tabular}



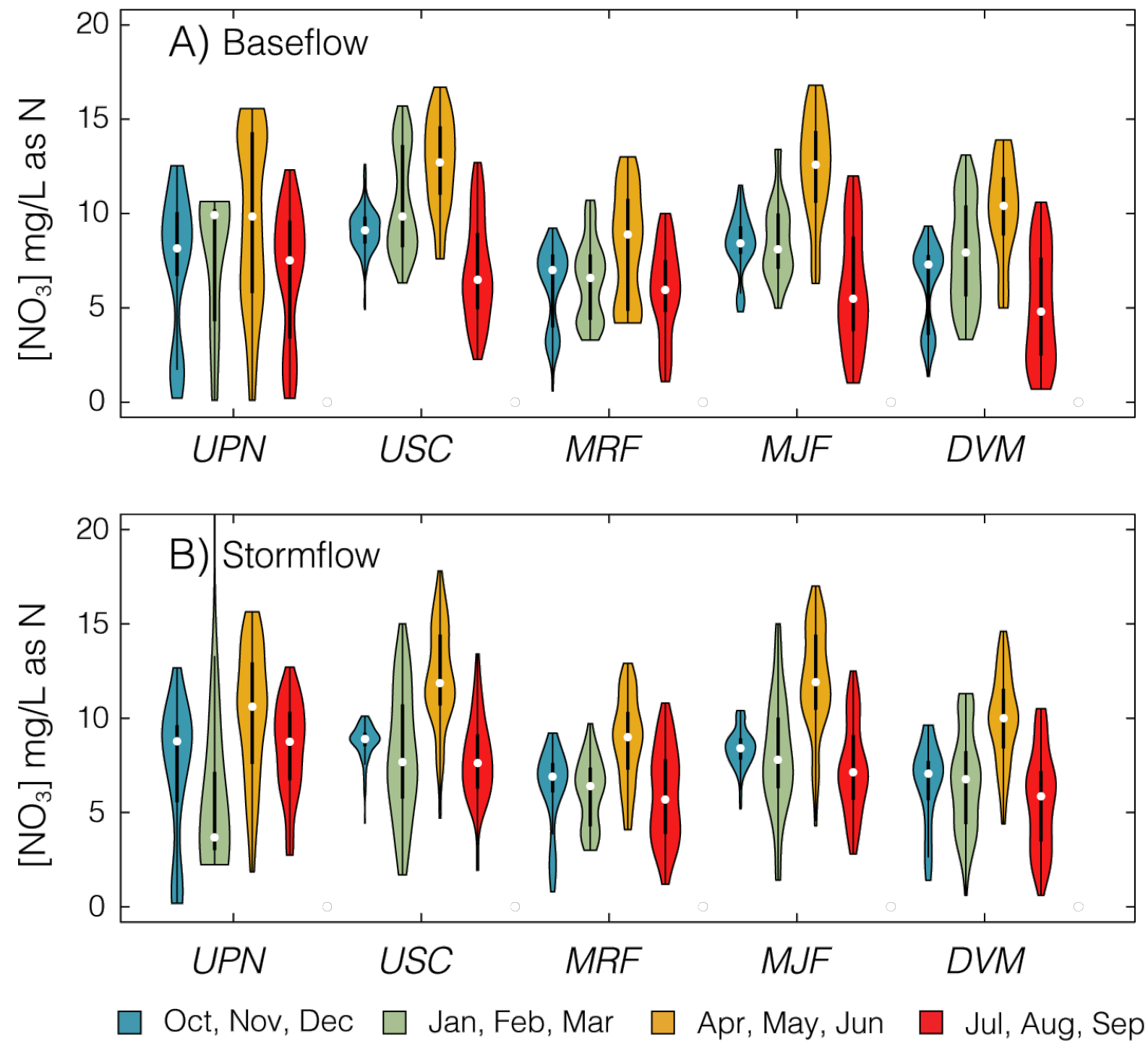

Figure $\mathrm{S} 2 \mathrm{NO}_{3}{ }^{-}$concentration by season for the five watersheds during baseflow (A) and stormflow periods (B). 

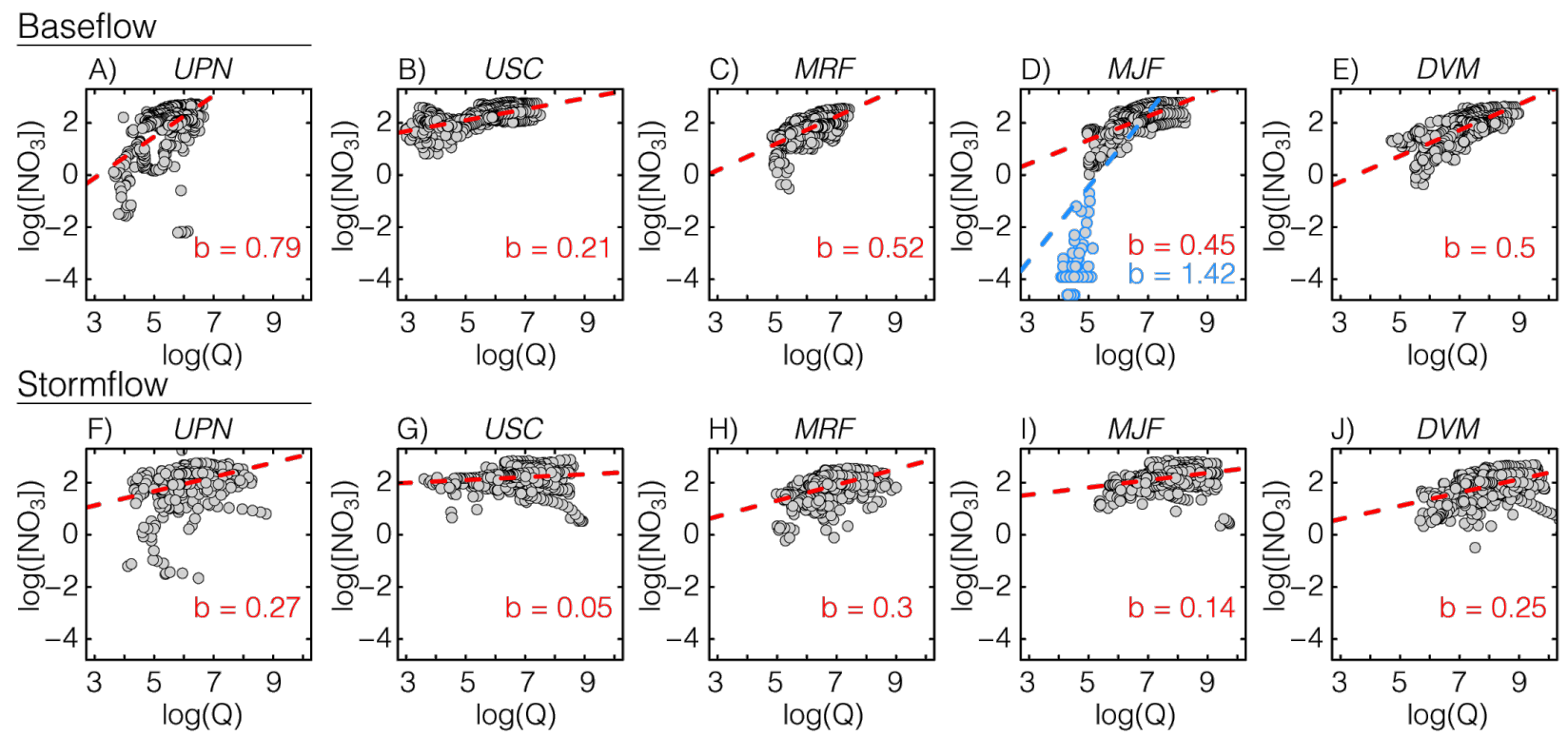

Figure S3 Concentration-discharge plots shown on log-log axes for five studied watersheds during baseflow (A-E) and stormflow (F-J), with the slope shown in red. Panel D shows the baseflow for $M J F$ with the low flow period from $7 / 27 / 17-10 / 19 / 17$ when $\left[\mathrm{NO}_{3}{ }^{-}\right]<0.5 \mathrm{mg} / \mathrm{L}$ included (blue) and excluded (red). All slopes are statistically significant $(p<0.01)$ 

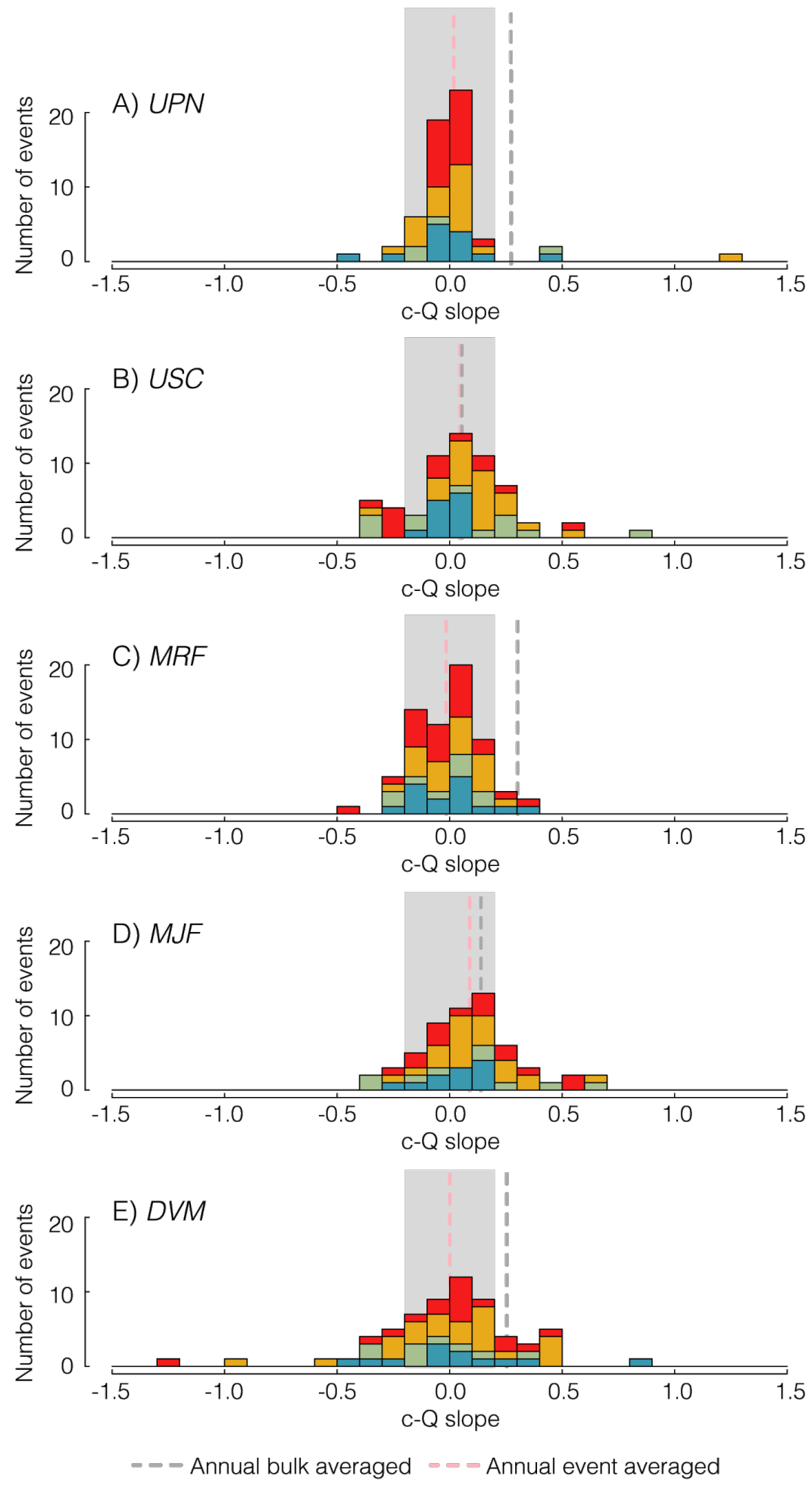

$\square$ Oct, Nov, Dec $\square$ Jan, Feb, Mar $\square$ Apr, May, Jun $\square$ Jul, Aug, Sep Figure S4 Histograms of individual event c-Q slope for each watershed colored by season. Gray dashed line shows the bulk stormflow c-Q slope and the pink dashed line shows the eventaveraged c-Q slope. Gray boxes delineate chemostatic behavior $(\mid \mathrm{c}-\mathrm{Q}$ slope $\mid<0.2)$. 
Table S3 - Nitrate concentration and load for each watershed during baseflow and stormflow

\begin{tabular}{|c|c|c|c|c|c|c|}
\hline \multirow[b]{2}{*}{ Watershed } & \multicolumn{2}{|c|}{ Baseflow+Stormflow } & \multicolumn{2}{|c|}{ Baseflow } & \multicolumn{2}{|c|}{ Stormflow } \\
\hline & $\begin{array}{c}\text { Nitrate } \\
\text { concentration }^{\mathrm{a}}\end{array}$ & $\begin{array}{l}\text { Cumulative } \\
\text { annual load }\end{array}$ & $\begin{array}{c}\text { Nitrate } \\
\text { concentration }\end{array}$ & $\begin{array}{l}\text { Cumulative } \\
\text { annual load }\end{array}$ & $\begin{array}{c}\text { Nitrate } \\
\text { concentration }\end{array}$ & $\begin{array}{l}\text { Cumulative } \\
\text { annual load }\end{array}$ \\
\hline & $\mathrm{mg} / \mathrm{L}$ as $\mathrm{N}$ & $\mathrm{kg}-\mathrm{N} / \mathrm{km}^{2} / \mathrm{yr}$ & $\mathrm{mg} / \mathrm{L}$ as $\mathrm{N}$ & $\mathrm{kg}-\mathrm{N} / \mathrm{km}^{2} / \mathrm{yr}$ & $\mathrm{mg} / \mathrm{L}$ as $\mathrm{N}$ & $\mathrm{kg}-\mathrm{N} / \mathrm{km}^{2} / \mathrm{yr}$ \\
\hline \multirow{2}{*}{$U P N$} & 8.27 & 3457 & 7.89 & 953 & 8.66 & 2507 \\
\hline & $(3.81)$ & $(940)$ & $(4.00)$ & $(162)$ & $(3.57)$ & (89) \\
\hline \multirow{2}{*}{ USC } & 9.56 & 3799 & 9.53 & 1108 & 9.58 & 2690 \\
\hline & $(3.09)$ & $(1133)$ & $(3.10)$ & $(492)$ & $(3.08)$ & $(675)$ \\
\hline \multirow{2}{*}{$M R F$} & 6.77 & 2222 & 6.56 & 813 & 7.03 & 1413 \\
\hline & $(2.51)$ & $(371)$ & $(2.43)$ & $(208)$ & $(2.59)$ & $(215)$ \\
\hline \multirow{2}{*}{$M J F$} & 9.11 & 4216 & 8.68 & 994 & 9.52 & 3222 \\
\hline & $(3.29)$ & $(768)$ & $(3.32)$ & $(269)$ & $(3.21)$ & $(674)$ \\
\hline \multirow{2}{*}{$D V M$} & 7.24 & 2829 & 6.94 & 799 & 7.55 & 2031 \\
\hline & $(3.07)$ & $(654)$ & $(3.08)$ & $(287)$ & $(3.02)$ & $(412)$ \\
\hline
\end{tabular}

${ }^{a}$ mean value is reported for nitrate concentration, numbers in parentheses are the standard deviation

${ }^{\mathrm{b}}$ mean annual cumulative load is reported, numbers in parentheses are the standard deviation 
Table S4 - Coefficient of variation (CV) values for c and Q during stormflow and baseflow

\begin{tabular}{ccccccc} 
& \multicolumn{3}{c}{ Stormflow } & \multicolumn{3}{c}{ Baseflow } \\
\cline { 2 - 7 } Watershed & $\mathrm{CV}_{\mathrm{c}}^{\mathrm{a}}$ & $\mathrm{CV}_{\mathrm{Q}}$ & $\mathrm{CV}_{\mathrm{c}} / \mathrm{CV}_{\mathrm{Q}}$ & $\mathrm{CV}_{\mathrm{c}}$ & $\mathrm{CV}_{\mathrm{Q}}$ & $\mathrm{CV}_{\mathrm{c}} / \mathrm{CV}_{\mathrm{Q}}$ \\
\hline$U P N$ & 0.412 & 1.058 & 0.390 & 0.506 & 0.603 & 0.840 \\
USC & 0.321 & 0.914 & 0.352 & 0.325 & 0.868 & 0.374 \\
$M R F$ & 0.369 & 0.842 & 0.438 & 0.370 & 0.558 & 0.664 \\
$M J F$ & 0.337 & 0.870 & 0.387 & 0.382 & 0.744 & 0.513 \\
$D V M$ & 0.401 & 0.886 & 0.452 & 0.444 & 0.785 & 0.565 \\
\hline
\end{tabular}

${ }^{\text {a }}$ Coefficient of variation $=$ standard deviation $/$ mean 
Table S5 Pearson correlation coefficient heat map for landscape attributes and nutrient export metrics, bold indicates $p<0.05$ and bold italtics indicates $p<0.01$

\begin{tabular}{|c|c|c|c|c|c|c|c|}
\hline & & & $\begin{array}{c}\text { Drainage } \\
\text { infrastruct. } \\
\text { density } \\
(\mathrm{km} / \mathrm{km} 2) \\
\end{array}$ & $\begin{array}{l}\text { Cropped } \\
\text { area within } \\
100 \mathrm{~m} \text { of } \\
\text { stream (\%) }\end{array}$ & $\begin{array}{l}\text { Cropped } \\
\text { area within } \\
1 \mathrm{~km} \text { of } \\
\text { stream (\%) }\end{array}$ & $\begin{array}{c}\text { Total } \\
\text { watershed } \\
\text { cropped } \\
\text { area }(\%)\end{array}$ & $\begin{array}{l}\text { Watershed } \\
\text { area }(\mathrm{km} 2)\end{array}$ \\
\hline \multirow{15}{*}{ Load } & \multirow{5}{*}{$\begin{array}{l}\text { Stormflow } \\
\text { +Baseflow }\end{array}$} & \multirow{5}{*}{$\begin{array}{c}\text { Complete Record } \\
\text { OND } \\
\text { JFM } \\
\text { AMJ } \\
\text { JAS } \\
\end{array}$} & 0.87 & 0.69 & -0.35 & 0.88 & -0.21 \\
\hline & & & 0.18 & 0.10 & -0.44 & 0.07 & -0.49 \\
\hline & & & 0.86 & 0.49 & -0.97 & 0.81 & 0.14 \\
\hline & & & 0.96 & 0.79 & -0.30 & 0.99 & -0.08 \\
\hline & & & 0.77 & 0.64 & -0.13 & 0.81 & -0.20 \\
\hline & & Complete Record & 0.84 & 0.61 & -0.32 & 0.86 & -0.14 \\
\hline & & OND & 0.34 & 0.23 & -0.49 & 0.24 & -0.48 \\
\hline & Stormflow & JFM & 0.89 & 0.42 & -0.86 & 0.89 & 0.28 \\
\hline & & AMJ & 0.94 & 0.74 & -0.26 & 0.99 & 0.00 \\
\hline & & JAS & 0.71 & 0.55 & -0.08 & 0.76 & -0.15 \\
\hline & & Complete Record & 0.87 & 0.95 & -0.47 & 0.79 & -0.56 \\
\hline & & OND & -0.14 & -0.16 & -0.31 & -0.25 & -0.46 \\
\hline & Baseflow & JFM & 0.58 & 0.49 & -0.92 & 0.45 & -0.15 \\
\hline & & AMJ & 0.94 & 0.95 & -0.42 & 0.89 & -0.37 \\
\hline & & JAS & 0.90 & 0.97 & -0.38 & 0.84 & -0.44 \\
\hline & & Complete Record & -0.76 & -0.80 & 0.08 & -0.80 & 0.03 \\
\hline & & OND & -0.53 & -0.54 & -0.25 & -0.65 & -0.26 \\
\hline & Stormflow & JFM & -0.54 & -0.32 & 0.68 & -0.43 & 0.38 \\
\hline & & AMJ & -0.79 & -0.50 & 0.13 & -0.89 & -0.41 \\
\hline & & JAS & -0.64 & -0.88 & -0.01 & -0.66 & 0.27 \\
\hline & & Complete Record & -0.93 & -0.89 & 0.31 & -0.93 & 0.16 \\
\hline & & OND & -0.42 & -0.49 & -0.41 & -0.56 & -0.23 \\
\hline$C-Q$ & Stormflow & JFM & -0.64 & -0.38 & 0.70 & -0.54 & 0.33 \\
\hline & & AMJ & -0.69 & -0.42 & 0.01 & -0.81 & -0.49 \\
\hline & & JAS & -0.73 & -0.88 & 0.57 & -0.59 & 0.73 \\
\hline & & Complete Record & -0.57 & -0.64 & -0.03 & -0.62 & -0.11 \\
\hline & & OND & -0.58 & -0.51 & -0.15 & -0.69 & -0.34 \\
\hline & Baseflow & JFM & -0.23 & -0.23 & 0.70 & -0.04 & 0.64 \\
\hline & & AMJ & -0.92 & -0.52 & 0.39 & -0.97 & -0.30 \\
\hline & & JAS & -0.56 & -0.65 & -0.07 & -0.62 & -0.09 \\
\hline
\end{tabular}

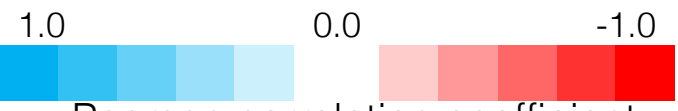

Pearson correlation coefficient 


\section{REFERENCES}

Garrett, J. D. (2019). The use of continuous water-quality time-series data to compute nutrient loadings for selected Iowa streams, 2008-17. Scientific Investigations Report. https://doi.org/10.3133/sir20195054

Jones, C. S., Schilling, K. E., Simpson, I. M., \& Wolter, C. F. (2018). Iowa Stream Nitrate, Discharge and Precipitation: 30-Year Perspective. Environmental Management, 62(4), 709720. https://doi.org/10.1007/s00267-018-1074-x

Pellerin, B. A., Bergamaschi, B. A., Gilliom, R. J., Crawford, C. G., Saraceno, J., Frederick, C. P., et al. (2014). Mississippi river nitrate loads from high frequency sensor measurements and regression-based load estimation. Environmental Science and Technology, 48(21), 12612-12619. https://doi.org/10.1021/es504029c

Zimmer, M. A., Pellerin, B., Burns, D. A., \& Petrochenkov, G. (2019). Temporal variability in nitrate-discharge relationships in large rivers as revealed by high-frequency data. Water Resources Research. https://doi.org/10.1029/2018WR023478 